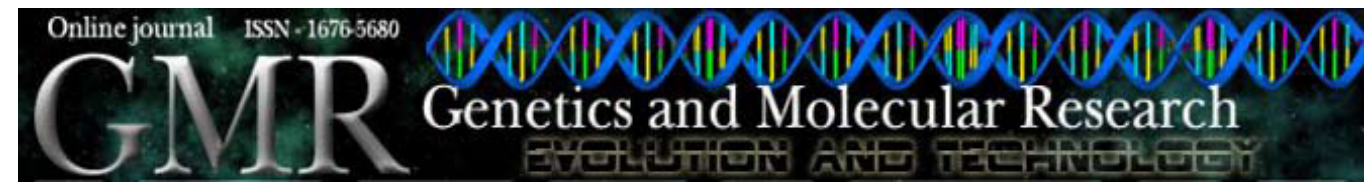

\title{
Association of SNPs on CAPN1 and CAST genes with tenderness in Nellore cattle
}

\author{
L.F.B. Pinto ${ }^{1}$, J.B.S. Ferraz ${ }^{1}$, F.V. Meirelles ${ }^{1}$, J.P. Eler ${ }^{1}$, F.M. Rezende ${ }^{1}$, \\ M.E. Carvalho ${ }^{1}$, H.B. Almeida ${ }^{2}$ and R.C.G. Silva ${ }^{2}$ \\ ${ }^{1}$ Grupo de Melhoramento Animal e Biotecnologia, \\ Departamento de Ciências Básicas, \\ Faculdade de Zootecnia e Engenharia de Alimentos, \\ Universidade de São Paulo, Pirassununga, SP, Brasil \\ ${ }^{2}$ Merial Saúde Animal Ltda., Campinas, SP, Brasil \\ Corresponding author: J.B.S. Ferraz \\ E-mail: jbferraz@usp.br
}

Genet. Mol. Res. 9 (3): 1431-1442 (2010)

Received April 19, 2010

Accepted May 22, 2010

Published July 20, 2010

DOI 10.4238/vol9-3gmr881

\begin{abstract}
We examined whether single-nucleotide polymorphisms (SNPs) in the calpain (CAPN) and calpastatin (CAST) genes, described from Bos primigenius taurus, are polymorphic in Nellore cattle. We also looked for a possible association of linkage disequilibrium of this polymorphism with tenderness of the longissimus dorsi muscle after 7, 14 and 21 days of postmortem aging in 638 purebred Nellore bulls. Meat tenderness was measured as Warner-Bratzler shear force. Additive and dominance effects were tested for SNPs of the three genotypic classes; the substitution effect was tested for SNPs with missing genotypic classes. Genotypic and gene frequencies were also calculated for the different SNPs. An increase in tenderness was observed from 7 to 21 days; the average values for shear force at 7, 14 and 21 days of aging were $5.92 \pm 0.06,4.92 \pm 0.05$, and 4.38 $\pm 0.04 \mathrm{~kg}$, respectively. All markers showed polymorphism, but there was no CC genotype for $C A P N 316$, and few animals showed the AA genotype for CAPN530. The alleles CAPN4751, UOGCAST1, and WSUCAST were found to have additive and dominance effects for shear force at 7, 14 and 21 days, while CAPN316 showed a substitution effect for shear force at
\end{abstract}


7 and 21 days. An additive-by-additive epistatic interaction was observed between CAPN4751 and markers on the CAST gene. In conclusion, these markers should be considered for use in breeding programs.

Key words: Bos primigenius indicus; Calpain; Calpastatin; TFAM; Meat quality

\section{INTRODUCTION}

Tenderness is an important beef trait, because it is associated with consumer satisfaction. However, tenderness is a complex trait for breeding programs, because evaluation also depends on how animals are slaughtered. Thus, molecular marker information can be of great usefulness for identification of animals with high genetic value for tenderness and the selection process can be conducted on younger animals, even before birth.

Polymorphisms on the calpain gene have been tested for many times in association studies for meat quality traits, mainly for tenderness and marbling (Smith et al., 2000; Page et al., 2002, 2004; White et al., 2005; Casas et al., 2005, 2006; Morris et al., 2006; Corva et al., 2007; Cheong et al., 2008). The calpain gene encodes the protease $\mu$-calpain that is involved in postmortem proteolysis (Koohmaraie, 1996), thus calpain is a good candidate gene for tenderness. Calpastatin is an inhibitor of $\mu$-calpain; therefore, it also has important function in the tenderization process. Polymorphisms on the calpastatin gene are associated with tenderness according to Casas et al. (2006) and Morris et al. (2006).

Several studies have been conducted on Bos primigenius taurus, but there are few studies on the association between single-nucleotide polymorphisms (SNPs) and tenderness on Bos primigenius indicus. Brahman cattle were evaluated by White et al. (2005) and Casas et al. $(2005,2006)$, but few significant effects were detected. White et al. (2005) reported an association between CAPN4751 and shear force at 7,14 and 21 days of postmortem aging. Validation of molecular markers is a very important step for use in breeding programs. Therefore, this study aims to validate SNPs on calpain and calpastatin genes, obtained in $B$. $p$. taurus, for Nellore cattle. The second objective was to carry out association tests between SNPs and tenderness of the longissimus dorsi muscle in Nellore bulls.

\section{MATERIAL AND METHODS}

\section{Population}

Phenotypic and genotypic information of 638 Nellore bulls of the Agropecuária CFM Ltda. were used. The animals were raised under pasture conditions until 18 months of age. From 18 months to slaughter, the bulls were fed in feedlots. The slaughter occurred between 22 and 26 months of age, on six different days, always in the mornings and after $16 \mathrm{~h}$ of fasting. All animals were identified for measurements and sample collection.

\section{Phenotypic traits}

After slaughter, the carcasses were sawed longitudinally in the middle. They were then 
chilled for $24 \mathrm{~h}$ at $2 \pm 1{ }^{\circ} \mathrm{C}$. After this period, three samples of longissimus dorsi were removed between the 12 and 13th ribs, always on the same carcass side. Samples were vacuum-packed and aged at $4 \pm 1{ }^{\circ} \mathrm{C}$ for 7,14 and 21 days. Tenderness was measured by Warner-Bratzler shear force, which determines the relative force required to pass a blunt blade through a section of meat. None of the samples were frozen.

\section{Genotyping and molecular markers}

The CAPN1 gene (GenBank accession Nos. AF248054 and AF252504) is located on chromosome 29, and four SNPs were used. The CAPN316 is a polymorphism in exon 9 of the CAPN1 gene, caused by a substitution of cytosine/guanine (C/G) (base 5709 of AF252504), that produces an amino acid substitution of alanine ( $\mathrm{C}$ allele) with glycine ( $\mathrm{G}$ allele) according to Page et al. (2002). The CAPN530 is an adenine/guanine (A/G) polymorphism in exon 14 of the gene (base 4558 of AF248054) that produces an amino acid substitution of isoleucine (A allele) with valine (G allele) according to Page et al. (2002). The CAPN4751 is a silent mutation located on intron 18 (base 6545 of AF248054) of CAPN1, caused by a substitution of cytosine/thymine $(\mathrm{C} / \mathrm{T})$ according to White et al. (2005). The CAPN4753 is a silent mutation located on intron 21 (base 8676 of AF248054) of CAPN1, caused by a substitution of adenine/ cytosine (A/C) according to Casas et al. (2005).

The CAST gene (GenBank accession No. AY008267) is located on chromosome 7, and two SNPs were used. The UOGCAST is a substitution of cytosine with guanine $(\mathrm{C} / \mathrm{G})$ located on intron 5 (base 282 of AY008267) according to Schenkel et al. (2006), while the WSUCAST is a substitution of cytosine with thymine in exon 3 (base 263 of AY008267) according to Garcia et al. (2006).

DNA samples were obtained from blood or hair follicles. Genotypes were obtained by DNA mass spectrometry (Sequenom iPlex ${ }^{\mathrm{TM}}$ Mass Spec), using Igenity ${ }^{\mathbb{B}}$ (Duluth, Georgia, USA). Polymerase chain reactions were carried out with 120 ng DNA, diluted in $25 \mu \mathrm{L}$, containing $0.2 \mu \mathrm{M}$ of each dNTP, $0.5 \mathrm{U}$ Taq DNA polymerase and $0.2 \mu \mathrm{M}$ of each oligonucleotide primer. A total of 32 cycles were performed for $1 \mathrm{~min}$ at $94^{\circ} \mathrm{C}, 30 \mathrm{~s}$ at $58^{\circ} \mathrm{C}$ and $1 \mathrm{~min}$ at $72^{\circ} \mathrm{C}$.

\section{Statistical analysis}

Gene and genotypic frequencies were obtained with the statistical analysis system (SAS, 2004). Associations between SNPs and traits were carried out with the QXPAK software (Pérez-Enciso and Misztal, 2004). A likelihood ratio test was conducted using a reduced model:

$$
Y_{i j}=\mu+G C_{i}+\alpha_{i j}(p H)+\beta_{i j}(S)+\lambda_{i j}(I)+e_{i j} \quad \text { (Equation 1) }
$$

where $Y_{i j}$ is the phenotypic value of the $\mathrm{j}^{\text {th }}$ animal in the $\mathrm{i}^{\text {th }}$ contemporary group, $\mu$ is the general mean of the trait, $G C_{i}$ is the fixed effect of $\mathrm{i}^{\text {th }}$ contemporary group, $\alpha_{i j}$ is the coefficient of the covariate $\mathrm{pH}$ of the carcass $24 \mathrm{~h}$ post mortem, $\beta_{i j}$ is the coefficient of the covariate age at slaughter, $\lambda_{i j}$ is the coefficient associated with the random effect of infinitesimal genetic value, and $e_{i j}$ is the random effect of the residual value.

The additive effect was tested using the full model: 


$$
Y_{i j}=\mu+G C_{i}+\alpha_{i j}(p H)+\beta_{i j}(S)+\lambda_{i j}(I)+\delta_{i j}(A)+e_{i j}
$$

where the new term is the coefficient $\delta_{i j}$ associated with the additive effect of the $\mathrm{j}^{\text {th }}$ animal in the $i^{\text {th }}$ contemporary group.

The dominance effect was tested using the full model:

$$
Y_{i j}=\mu+G C_{i}+\alpha_{i j}(p H)+\beta_{i j}(S)+\lambda_{i j}(I)+\gamma_{i j}(D)+e_{i j} \quad \text { (Equation 3) }
$$

where the new term is the coefficient $\gamma_{i j}$ associated with the dominance effect of the $\mathrm{j}^{\text {th }}$ animal in the $\mathrm{i}^{\text {th }}$ contemporary group. A likelihood ratio test without infinitesimal genetic value on full and reduced models was also carried out to compare the results.

Regression was adjusted in the SAS (2004) to test for the epistatic effect. To test for the additive effect, the dummy variables were generated with values $(1,0$ and -1$)$ for AA, Aa and aa genotypes, respectively. To estimate the dominance effect, dummy variables were generated with values $(0,1$ and 0$)$ for AA, Aa and aa genotypes, respectively. The model adjusted for all traits was:

$$
\begin{aligned}
& Y_{i j}=\mu+G C_{i}+\sigma_{i j}(p H)+\beta_{i}(S)+\delta_{i j}\left(A_{1}\right)+\lambda_{i j}\left(D_{1}\right)+\phi_{i j}\left(A_{2}\right)+\gamma_{i j}\left(D_{2}\right) \\
& +v_{i j}\left(A_{1} A_{2}\right)+\theta_{i j}\left(A_{1} D_{2}\right)+\vartheta_{i j}\left(D_{1} A_{2}\right)+\sigma_{i j}\left(D_{1} D_{2}\right)+e_{i j}
\end{aligned}
$$

with marginal effects of two SNPs more epistatic interaction between marginal effects.

\section{RESULTS AND DISCUSSION}

\section{Descriptive analysis}

Shear force at 14 and 21 days of postmortem aging (Table 1) is similar to that described by Bonilha et al. (2008), which found 4.56 and 4.53 for selected and non-selected Nellore, respectively. It is important to note that there is large variance for this trait in Nellore cattle. In the sample size, there are very tender steaks (shear force values of less than two), but there are also very tough steaks (shear force values of more than nine). Therefore, tenderness is a trait with great potential for selection programs in the Nellore breed.

\begin{tabular}{lccccr}
\multicolumn{5}{r}{ Table 1. Descriptive analysis for tenderness after 7 (T7D), 14 (T14D) and 21 (T21D) days of postmortem aging. } \\
\hline Trait & $\mathrm{N}$ & Average (SE) & Minimum & Maximum & CV \\
\hline T7D & 630 & $5.92^{\mathrm{a}}(0.06)$ & 1.82 & 9.99 & 24.33 \\
T14D & 629 & $4.92^{\mathrm{b}}(0.05)$ & 1.38 & 8.39 & 25.83 \\
T21D & 633 & $4.38^{\mathrm{c}}(0.04)$ & 1.61 & 7.73 & 25.58 \\
\hline
\end{tabular}

$\mathrm{N}=$ sample size analyzed; $\mathrm{SE}=$ standard error; $\mathrm{CV}=$ coefficient of variation. Average values followed by different letters are significantly different by the Tukey test with a significance level of $5 \%$.

\section{Gene and genotypic frequencies}

The first objective of this study was to test whether SNPs associated with tenderness in $B$. p. taurus are polymorphic in Nellore cattle. The results showed that the markers 
CAPN4751, CAPN4753, UOGCAST, WSUCAST are polymorphic in Nellore, because no fixed alleles were observed and the allele frequency for all markers was above 1\% (Table 2). However, for CAPN316 and CAPN530 few CC and AA animals were observed, respectively, and the frequencies of alleles $\mathrm{C}$ and $\mathrm{A}$ were below $1 \%$. Reduced polymorphisms for the markers CAPN316 and CAPN530 in B. p. indicus have been reported in other studies. Casas et al. (2005) analyzed 460 Brahman cattle and found no CC genotype at CAPN316, while for CAPN530 the $\mathrm{G}$ allele was fixed. In B. p. taurus, the markers CAPN530 and CAPN316 are more polymorphic as can be observed in Page et al. (2002, 2004), Morris et al. (2006), and Corva et al. (2007). The additive effect is difficult to estimate with reduced genotypic frequencies, as it is obtained through the contrast between homozygotes, because the phenotypic average might have been underestimated or overestimated for the rare genotype.

\begin{tabular}{|c|c|c|c|c|c|}
\hline \multirow{2}{*}{$\begin{array}{l}\text { Markers } \\
C A P N 4753\end{array}$} & \multicolumn{2}{|c|}{ Gene frequencies } & \multicolumn{3}{|c|}{ Genotypic frequencies } \\
\hline & A - 0.66 & $C-0.34$ & $\mathrm{f}(\mathrm{AA})=51.0$ & $f(A C)=30.0$ & $f(C C)=19.0$ \\
\hline CAPN4751 & $C-0.18$ & $\mathrm{~T}-0.82$ & $\mathrm{f}(\mathrm{CC})=3.2$ & $f(C T)=29.3$ & $f(T T)=67.5$ \\
\hline CAPN530 & $\mathrm{G}-0.92$ & $\mathrm{~A}-0.08$ & $\mathrm{f}(\mathrm{GG})=84.7$ & $\mathrm{f}(\mathrm{GA})=15.0$ & $f(A A)=0.3$ \\
\hline$C A P N 316$ & $C-0.08$ & G - 99.2 & $f(C C)=0.0$ & $\mathrm{f}(\mathrm{CG})=1.6$ & $f(G G)=98.4$ \\
\hline UOGCASTI & $C-0.63$ & $\mathrm{G}-0.37$ & $f(C C)=39.7$ & $\mathrm{f}(\mathrm{CG})=47.1$ & $f(G G)=13.2$ \\
\hline WSUCAST & $C-0.37$ & $\mathrm{~T}-0.63$ & $f(C C)=13.4$ & $f(C T)=47.3$ & $\mathrm{f}(\mathrm{TT})=39.3$ \\
\hline
\end{tabular}

CAPN4751 was the first marker on the CAPN1 gene to show significant association with meat tenderness in both B. p. taurus and B. p. indicus (White et al., 2005). In the present study, CAPN4751 was more informative than in Brahman cattle, because we found all three genotypic classes in Nellore, while in Brahman cattle the CC genotype was not observed by White et al. (2005) or Casas et al. (2006). Of all markers on the CAPN1 gene studied here, CAPN4753 showed the best segregation (Table 2). All genotypic classes were observed, and the class with the fewest animals (CC) represents 19\% of the total sample. Casas et al. (2005) reported good segregation of this marker in Brahman cattle, with a frequency of $8.8 \%$ for the CC genotype. For testing the epistatic effect it is important that all genotypic classes be present in the sample size and that all classes have a large number of animals, because this allows the mean value in all combinations of genotypic classes to be estimated with good accuracy. We expect that the epistatic effect estimated between CAPN4753 and SNPs on the CAST gene will present good accuracy.

The markers on the CAST gene showed higher quality of polymorphism than SNPs on the CAPN1 gene (Table 2). There is a sufficient number of homozygotes for estimating accurate additive and dominance effects for UOGCAST1 and WSUCAST. Those markers also showed similar gene and genotypic frequencies, which may be a consequence of linkage disequilibrium between them, that would be inherited like a haplotype. Schenkel et al. (2006) found gene frequencies ranging from 36.4 (Simmental) to 68.8 (Charolais) for the $\mathrm{C}$ allele at UOGCAST1 in several B. $p$. taurus-influenced cattle, but across the breed they found average values similar to the present study.

\section{Association analysis}

\section{CAPN4751}

CAPN4751 was associated with tenderness at 7, 14 and 21 days of postmortem aging, because the contrast between CC and TT genotypes was significant. The favorable allele 
was $\mathrm{C}$ and the contrasts (CC-TT) were estimated at $-0.30,-0.27$ and $-0.34 \mathrm{~kg}$ of shear force for longissimus dorsi muscle steaks at 7,14 and 21 days of aging, respectively (Table 3 ). The dominance effect was found at CAPN4751 for all aging periods and CC and CT genotypes were more tender than the TT genotype. Results observed in Nellore cattle confirm previous studies that documented the effect of SNP CAPN4751 on tenderness in B. p.taurus, B. p. indicus and crossbreed populations as can be observed in White et al. (2005), Casas et al. (2006) and Barendse et al. (2007). Therefore, CAPN4751 is really an important SNP on the CAPN1 gene, because it explains the functional variation for tenderness in many bovine subspecies.

\begin{tabular}{llcrrr}
\multicolumn{5}{c}{ Table 3. Association among SNPs and tenderness after 7 (T7D), 14 (T14D) and 21 (T21D) days of postmortem aging. } \\
\hline Trait & Marker & Type of effect & Effect (SE) & LRT & Nominal P value \\
\hline T7D & CAPN4751 & A & $-0.30(0.10)$ & 8.33 & 0.0039 \\
& & D & $-0.38(0.12)$ & 9.44 & 0.0021 \\
T7D & UOGCASTI & $\mathrm{A}$ & $-0.17(0.08)$ & 4.04 & 0.0443 \\
& & $\mathrm{D}$ & $0.25(0.11)$ & 5.00 & 0.0253 \\
T7D & WSUCAST & $\mathrm{A}$ & $0.16(0.08)$ & 3.84 & 0.0499 \\
& & $\mathrm{D}$ & $0.24(0.11)$ & 4.85 & 0.0276 \\
T14D & CAPN4751 & $\mathrm{A}$ & $-0.27(0.09)$ & 9.44 & 0.0021 \\
& & $\mathrm{D}$ & $-0.28(0.11)$ & 6.72 & 0.0095 \\
T14D & UOGCASTI & $\mathrm{A}$ & $-0.21(0.07)$ & 8.43 & 0.0036 \\
T14D & WSUCAST & $\mathrm{A}$ & $0.21(0.07)$ & 8.41 & 0.0037 \\
T21D & CAPN4751 & $\mathrm{A}$ & $-0.34(0.08)$ & 17.56 & 0.00003 \\
& & $\mathrm{D}$ & $-0.34(0.09)$ & 12.76 & 0.0003 \\
T21D & UOGCASTI & $\mathrm{A}$ & $-0.25(0.06)$ & 14.16 & 0.0002 \\
& & $\mathrm{D}$ & $0.21(0.09)$ & 5.43 & 0.0198 \\
T21D & WSUCAST & $\mathrm{A}$ & $0.23(0.06)$ & 12.21 & 0.0005 \\
& & $\mathrm{D}$ & $0.18(0.08)$ & 4.27 & 0.0388 \\
\hline
\end{tabular}

$\mathrm{SE}=$ standard error; $\mathrm{LTR}=$ likelihood ratio test $\mathrm{A}=$ additive effect $\mathrm{D}=$ dominance effect.

Adjustment of the infinitesimal genetic value (Table 4) for estimating additive and dominance effects at CAPN4751 showed results similar to previous analysis (Table 3 ). However, the additive effect across aging rose with estimates of $-0.26,-0.28$ and -0.33 for contrast (CC-TT) at 7,14 and 21 days, respectively. This increase in tenderness is believed to be due to increased postmortem aging. Thus, the results in the infinitesimal genetic model seem to be more appropriate.

\begin{tabular}{|c|c|c|c|c|c|}
\hline Trait & Marker & Type of effect & Effect (SE) & LRT & Nominal $\mathrm{P}$ value \\
\hline \multirow[t]{2}{*}{ T7D } & CAPN4751 & A & $-0.26(0.11)$ & 5.82 & 0.0158 \\
\hline & & D & $-0.32(0.12)$ & 6.57 & 0.0104 \\
\hline \multirow[t]{2}{*}{ T14D } & CAPN4751 & A & $-0.28(0.09)$ & 9.20 & 0.0024 \\
\hline & & D & $-0.26(0.11)$ & 5.75 & 0.0165 \\
\hline T14D & UOGCASTI & A & $-0.18(0.07)$ & 6.15 & 0.0132 \\
\hline T14D & WSUCAST & A & $0.18(0.07)$ & 6.03 & 0.0141 \\
\hline \multirow[t]{2}{*}{ T21D } & CAPN4751 & A & $-0.33(0.08)$ & 15.80 & 0.00007 \\
\hline & & D & $-0.32(0.10)$ & 10.70 & 0.0011 \\
\hline \multirow[t]{2}{*}{ T21D } & UOGCASTI & A & $-0.23(0.07)$ & 11.57 & 0.0007 \\
\hline & & D & $0.18(0.09)$ & 4.29 & 0.0383 \\
\hline T21D & WSUCAST & A & $0.21(0.07)$ & 9.83 & 0.0017 \\
\hline
\end{tabular}

$\mathrm{SE}=$ standard error; $\mathrm{LRT}=$ likelihood ratio test; $\mathrm{A}=$ additive effect; $\mathrm{D}=$ dominance effect.

\section{CAPN4753}

In the present study, it was not possible to find an association between $C A P N 4753$ and 
tenderness of longissimus dorsi muscle. The lowest likelihood ratio test $\mathrm{P}$ value for the additive effect was observed when we used shear force on meat with 21 days of aging (nominal $\mathrm{P}$ value $=$ 0.0699 ) and a model without infinitesimal genetic value. A similar result was observed by Casas et al. (2005) who also found no association between CAPN4753 and meat traits in Brahman cattle. Increasing the sample size can result in a significant effect $(\mathrm{P}<0.05)$, but due to great segregation of this marker we think that this hypothesis is much reduced. Nevertheless, CAPN4753 is useful in association studies that use other complex traits in Nellore cattle due to their good segregation. For instance, Casas et al. (2005) found an association of CAPN4753 with hump height $(\mathrm{P}=0.003)$.

\section{UOGCAST1}

Calpastatin has an important function in the tenderization process through regulating calpain activity (Koohmaraie, 1996). The CAST gene (GenBank accession No. AY008267) is located on chromosome 7 and some polymorphisms on this gene have been recently studied (Casas et al., 2006; Schenkel et al., 2006; Barendse et al., 2007). The SNP UOGCAST1 is a substitution of cytosine with guanine $(\mathrm{C} / \mathrm{G})$ located on intron 5 of the CAST gene (base 282 of AY008267), as described by Schenkel et al. (2006). This marker is currently used as the Igenity TenderGene marker test and it was associated with tenderness in a crossbreed of Angus, Charolais, Limousin, and Simmental by Shenkel et al. (2006). However, the present study is the first to report an association of a marker on the CAST gene with shear force in purebred B. p. indicus-influenced cattle. In Nellore cattle, we observed an additive effect of the UOGCAST1 in all aging periods (Table 3), and a significant dominance effect at 7 and 21 days of aging. This marker showed the $\mathrm{C}$ allele favorable and the $\mathrm{G}$ allele dominant, therefore, animals with the CC genotype have more tender meat than CG or GG animals. The contrast (CC- GG) for shear force showed $-0.17,-0.21$ and -0.25 of additive effect for 7,14 and 21 days of aging, respectively. When we adjust the infinitesimal genetic value for analyzing UOGCAST1, no additive or dominant effect at 7 days of postmortem aging was observed (Table 4), but the previous results (Table 3 ) remained significant. An increase in sample size can help to show if this effect also is present during the initial aging process.

\section{WSUCAST}

The SNP WSUCAST is also located on the CAST gene. However, this marker is a substitution of cytosine with thymine in exon 3 (base 263 of AY008267) (Garcia et al., 2006). The base 263 is much closer to the base 283 for UOGCAST1 and therefore those markers may be inherited like haplotypes. The results of the present study for polymorphisms and association tests confirm this hypothesis, because the values obtained for UOGCAST and WSUCAST were very similar.

\section{Substitution effect test}

Additive or dominance effects were not estimated for CAPN316 because the CC genotype was not found. CAPN530 was the only marker with no additive or dominance effects on tenderness, maybe a consequence of reduced AA genotype (only 2 animals). Thus, for CAPN316 and CAPN530 the substitution effect was estimated.

The substitution effect was significant only for shear force measured at 14 days of 
aging at $C A P N 316$ (Table 5). CAPN316 was associated with beef tenderness by Page et al. (2004), White et al. (2005), Morris et al. (2006), and Corva et al. (2007) in B. p. taurusinfluenced cattle, but also by Casas et al. (2005) in an analysis of tenderness score in $B . p$. indicus (Brahman cattle). In all above studies the $\mathrm{C}$ allele was favorable for more tender steaks, which is similar to the present study. The contrast (CG-GG) showed values of -0.71 , -1.1 , and $-0.69 \mathrm{~kg}$ of shear force at 7, 14 and 21 days of postmortem aging, respectively. The contrasts observed at 7 and 21 days of aging may be significant too, but the reduced number of CG animals produced high standard errors for CG genotype values. Due to the reduced frequency of the $\mathrm{C}$ allele in Nellore cattle this marker could be of great importance in this breed, because increasing the favorable allele frequency can result in more tender steaks.

Table 5. Substitution effect at $C A P N 316$ for tenderness measured by shear force for longissimus dorsi muscle at
\begin{tabular}{lccc}
$7(\mathrm{~T} 7 \mathrm{D}), 14(\mathrm{~T} 14 \mathrm{D})$ and $21(\mathrm{~T} 21 \mathrm{D})$ days of postmortem aging. \\
\hline Trait & Genotype & $\mathrm{N}$ & $\mathrm{WBSF} \pm \mathrm{SE}(\mathrm{kg})$ \\
\hline T7D & CG & 10 & $5.02 \pm 0.53$ \\
& GG & 627 & $5.73 \pm 0.09$ \\
T14D & G & 10 & $3.63 \pm 0.45^{*}$ \\
& GG & 627 & $4.73 \pm 0.08^{*}$ \\
T21D & GG & 10 & $3.57 \pm 0.42$ \\
& GG & 627 & $4.26 \pm 0.07$ \\
\hline
\end{tabular}

$\mathrm{N}=$ sample size; $\mathrm{WBSF}=$ Warner-Bratzler shear force; $\mathrm{SE}=$ standard error. *Statistically significant.

\section{Epistasis effects}

Epistatic models are very complex, but extremely important to evaluate the variance of quantitative traits. The problem is how to test several parameters in the model with reduced sample size. Here we implement epistatic models to evaluate possible interaction among SNPs on the CAPN1 gene (CAPN4751 and CAPN4753) and SNPs on the CAST gene (UOGCAST and WSUCAST). Additive-by-additive, additive-by-dominance, dominance-byadditive, and dominance-by-dominance effects were estimated and tested jointly and separately. When testing these effects, it is fundamental that all genotypic classes have a large number of animals. In Table 6, it is evident that the combinations of the marker CAPN4753 with UOGCAST and WSUCAST have a sufficient number of animals for estimating the least square mean values for all genotypic classes. However, for the marker CAPN4751, the proportion of animals with the $\mathrm{CC}$ genotype is reduced, thus the combination of this genotype with genotypes of markers UOGCAST (CCCC $=4$ animals, $\mathrm{CCCG}=11$ animals, and CCGG $=5$ animals $)$ and $W S U C A S T$ (CCCC $=5$ animals, CCCT $=10$ animals, CCTT $=5$ animals $)$ was less frequent (Table 7).

\begin{tabular}{|c|c|c|c|c|}
\hline & & \multicolumn{3}{|c|}{ CAPN4753 } \\
\hline & & $\overline{\mathrm{AA}}$ & $\mathrm{AC}$ & $\mathrm{CC}$ \\
\hline \multirow[t]{3}{*}{ UOGCAST } & $\mathrm{CC}$ & 50 & 58 & 109 \\
\hline & CG & 43 & 84 & 148 \\
\hline & GG & 11 & 27 & 34 \\
\hline \multirow[t]{3}{*}{ WSUCAST } & $\mathrm{CC}$ & 12 & 28 & 34 \\
\hline & CT & 43 & 86 & 149 \\
\hline & TT & 50 & 59 & 109 \\
\hline
\end{tabular}


Table 7. Frequency of combination of marker CAPN4751 with UOGCAST and WSUCAST.

\begin{tabular}{lrrrr}
\hline & & & CAPN4751 & CT \\
\cline { 3 - 5 } & & CC & 72 & 162 \\
UOGCAST & CC & 4 & 85 & 195 \\
& CG & 11 & 23 & 51 \\
\multirow{3}{*}{ WSUCAST } & 5 & 23 & 53 \\
& GG & 5 & 84 & 201 \\
& CC & 10 & 74 & 160 \\
\hline
\end{tabular}

We hoped to find an epistatic interaction between SNPs on CAPN1 and CAST genes, due to the biological mechanism of action of $\mu$-calpain and calpastatin enzymes, described in Koohmaraie (1996). However, only two significant interactions were detected in the present study (Table 8), both for shear force measured at 14 days of aging. CAPN4751 has significant additive-by-additive interaction with the markers WSUCAST and UOGCAST. Additive-by-additive interaction between CAPN4751 and markers on the CAST gene are plotted in Figures 1 and 2. The graphics are very similar, and once more demonstrate that WSUCAST and UOGCAST are linked and are probably heredity like haplotypes.

\begin{tabular}{|c|c|c|c|c|c|c|c|c|c|c|c|c|c|c|}
\hline Trait & Marker 1 & Marker 2 & a1 & SE & $\mathrm{P}$ & a2 & SE & $\mathrm{P}$ & $\mathrm{d} 1$ & SE & $\mathrm{P}$ & $\mathrm{d} 2$ & SE & $\mathrm{P}$ \\
\hline T7D & CAPN4751 & UOGCAST & 0.07 & 0.25 & 0.78 & 0.07 & 0.25 & 0.79 & -0.05 & 0.30 & 0.87 & 0.25 & 0.33 & 0.45 \\
\hline T14D & CAPN4751 & UOGCAST & -0.02 & 0.21 & 0.92 & -0.71 & 0.21 & 0.00 & 0.15 & 0.26 & 0.56 & -0.05 & 0.28 & 0.85 \\
\hline T21D & CAPN4751 & $U O G C A S T$ & -0.09 & 0.19 & 0.63 & -0.01 & 0.20 & 0.95 & -0.01 & 0.24 & 0.98 & -0.14 & 0.26 & 0.60 \\
\hline T7D & CAPN4751 & WSUCAST & 0.08 & 0.23 & 0.74 & -0.08 & 0.24 & 0.74 & -0.06 & 0.29 & 0.83 & 0.23 & 0.33 & 0.49 \\
\hline T14D & CAPN4751 & WSUCAST & 0.08 & 0.20 & 0.71 & 0.62 & 0.20 & 0.00 & 0.06 & 0.25 & 0.80 & -0.18 & 0.28 & 0.53 \\
\hline $\mathrm{T} 21 \mathrm{D}$ & CAPN4751 & WSUCAST & -0.05 & 0.18 & 0.79 & -0.03 & 0.18 & 0.86 & -0.01 & 0.23 & 0.95 & -0.24 & 0.26 & 0.36 \\
\hline T7D & $C A P N 4753$ & $U O G C A S T$ & 0.17 & 0.14 & 0.23 & -0.03 & 0.14 & 0.83 & 0.04 & 0.22 & 0.86 & 0.43 & 0.19 & 0.02 \\
\hline T14D & CAPN4753 & UOGCAST & 0.13 & 0.12 & 0.30 & -0.14 & 0.12 & 0.26 & -0.28 & 0.19 & 0.15 & -0.04 & 0.16 & 0.79 \\
\hline T21D & CAPN4753 & UOGCAST & 0.10 & 0.11 & 0.37 & -0.24 & 0.11 & 0.03 & -0.23 & 0.17 & 0.18 & 0.16 & 0.15 & 0.29 \\
\hline $\mathrm{T} 7 \mathrm{D}$ & CAPN4753 & WSUCAST & 0.16 & 0.14 & 0.26 & -0.01 & 0.14 & 0.92 & 0.09 & 0.22 & 0.69 & 0.43 & 0.19 & 0.02 \\
\hline T14D & CAPN4753 & WSUCAST & 0.08 & 0.12 & 0.48 & 0.10 & 0.12 & 0.41 & -0.23 & 0.19 & 0.22 & 0.03 & 0.16 & 0.84 \\
\hline T21D & CAPN4753 & WSUCAST & 0.07 & 0.11 & 0.49 & 0.20 & 0.11 & 0.06 & -0.18 & 0.17 & 0.30 & 0.19 & 0.15 & 0.20 \\
\hline Trait & Marker 1 & Marker 2 & $\mathrm{a} 1 \mathrm{a} 2$ & SE & $\mathrm{P}$ & $\mathrm{a} 1 \mathrm{~d} 2$ & SE & $\mathrm{P}$ & $\mathrm{d} 1 \mathrm{a} 2$ & $\mathrm{SE}$ & $\mathrm{P}$ & $\mathrm{d} 1 \mathrm{~d} 2$ & $\mathrm{SE}$ & $\mathrm{P}$ \\
\hline T7D & $C A P N 4751$ & UOGCAST & -0.03 & 0.25 & 0.91 & -0.17 & 0.33 & 0.60 & -0.36 & 0.31 & 0.24 & -0.31 & 0.41 & 0.44 \\
\hline T14D & CAPN4751 & UOGCAST & -0.73 & 0.21 & 0.00 & -0.34 & 0.28 & 0.23 & 0.28 & 0.26 & 0.28 & -0.18 & 0.35 & 0.61 \\
\hline T21D & CAPN4751 & UOGCAST & 0.07 & 0.20 & 0.73 & -0.34 & 0.26 & 0.19 & -0.36 & 0.24 & 0.13 & 0.09 & 0.32 & 0.77 \\
\hline T7D & CAPN4751 & WSUCAST & 0.00 & 0.24 & 0.99 & -0.20 & 0.33 & 0.54 & 0.36 & 0.29 & 0.22 & -0.31 & 0.41 & 0.44 \\
\hline T14D & CAPN4751 & WSUCAST & 0.63 & 0.20 & 0.00 & -0.49 & 0.28 & 0.08 & -0.19 & 0.25 & 0.44 & -0.03 & 0.35 & 0.93 \\
\hline T21D & CAPN4751 & WSUCAST & -0.11 & 0.18 & 0.56 & -0.46 & 0.26 & 0.08 & 0.37 & 0.23 & 0.10 & 0.11 & 0.32 & 0.74 \\
\hline T7D & CAPN4753 & $U O G C A S T$ & -0.05 & 0.14 & 0.70 & 0.01 & 0.19 & 0.97 & -0.07 & 0.22 & 0.74 & -0.49 & 0.30 & 0.11 \\
\hline T14D & CAPN4753 & UOGCAST & 0.06 & 0.12 & 0.60 & -0.13 & 0.16 & 0.44 & 0.00 & 0.19 & 0.99 & 0.34 & 0.26 & 0.19 \\
\hline $\mathrm{T} 21 \mathrm{D}$ & CAPN4753 & UOGCAST & 0.00 & 0.11 & 0.97 & 0.14 & 0.15 & 0.36 & 0.12 & 0.17 & 0.48 & 0.00 & 0.24 & 0.99 \\
\hline T7D & CAPN4753 & WSUCAST & 0.01 & 0.14 & 0.94 & 0.01 & 0.19 & 0.96 & 0.16 & 0.22 & 0.47 & -0.48 & 0.30 & 0.11 \\
\hline T14D & CAPN4753 & WSUCAST & -0.10 & 0.12 & 0.42 & -0.07 & 0.16 & 0.65 & 0.06 & 0.19 & 0.74 & 0.27 & 0.26 & 0.30 \\
\hline T21D & CAPN4753 & WSUCAST & -0.03 & 0.11 & 0.79 & 0.17 & 0.15 & 0.23 & -0.09 & 0.17 & 0.58 & -0.10 & 0.23 & 0.66 \\
\hline
\end{tabular}

a1 and a $2=$ marginal additive effects of markers 1 and 2 , respectively; $\mathrm{d} 1$ and $\mathrm{d} 2=$ marginal dominance effects of markers 1 and 2, respectively; a1a2 = interaction between marginal additive effects of markers 1 and 2; a1d2 = interaction between marginal additive effect of marker 1 and marginal dominance effect of marker 2; $\mathrm{d} 1 \mathrm{a} 2=$ interaction between marginal dominance effect of marker 1 and marginal additive effect of marker 2; $\mathrm{d} 1 \mathrm{~d} 2=$ interaction between marginal dominance effects of markers 1 and 2 . SE $=$ standard error; T7D, T14D, $\mathrm{T} 21 \mathrm{D}=$ tenderness after 7,14 and 21 days of postmortem aging. 


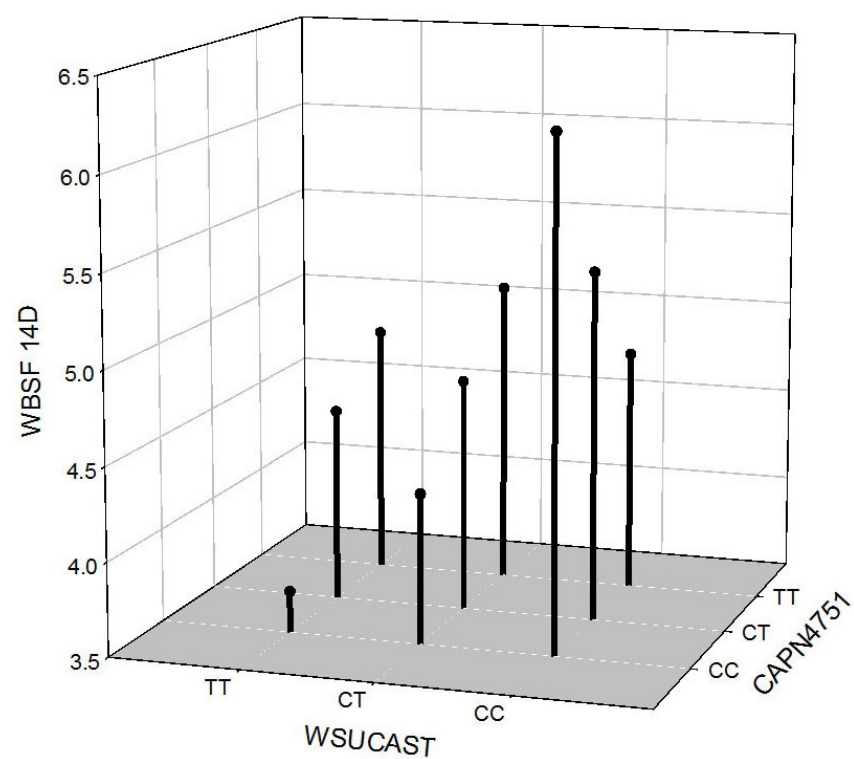

Figure 1. 3-D plot of genotype class combinations of CAPN4751 and WSUCAST markers. WBSF = WarnerBratzler shear force.

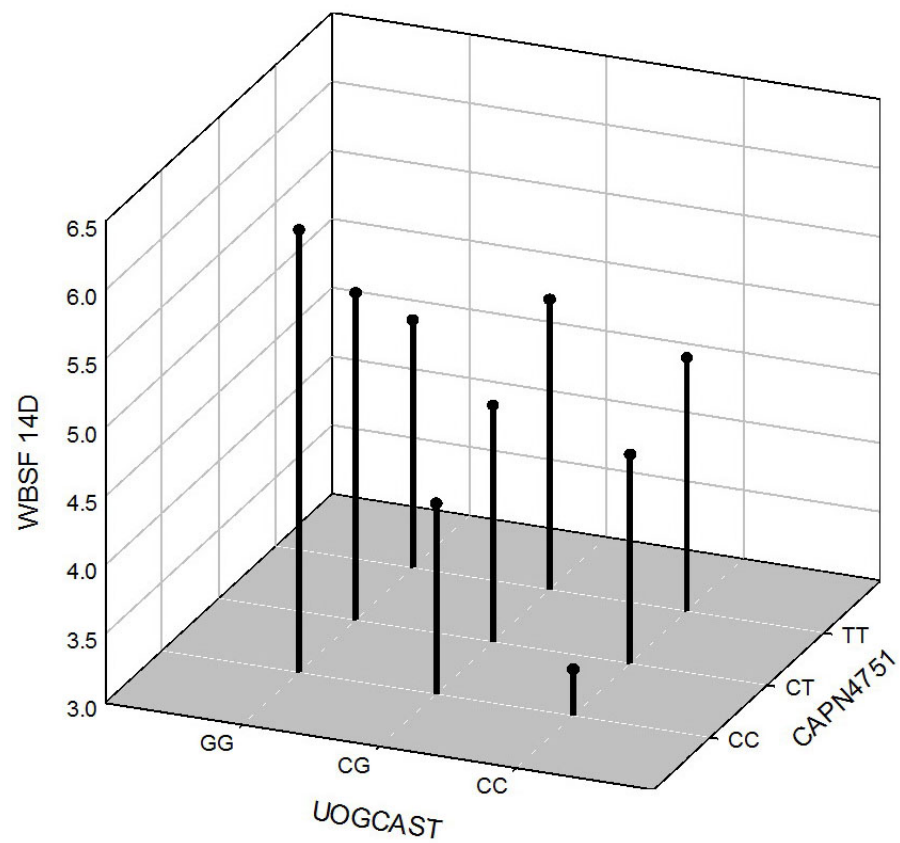

Figure 2. 3-D plot of genotype class combinations of CAPN4751 and UOGCAST markers. WBSF = WarnerBratzler shear force. 
The additive effect of WSUCAST and UOGCAST is observed only when the genotypic class of CAPN4751 is different from TT. When CAPN4751 is TT the markers on the CAST gene present only the dominance effect. Another important piece of information from these graphics is that the favorable allele for CAPN4751 can be T or C, depending on the genotypic class of markers on the CAST gene. When WSUCAST or UOGCAST are CC homozygous the favorable allele on CAPN4751 is T. Therefore, it is necessary that there be at least one T allele in WSUCAST or UOGCAST for the favorable allele at CAPN4751 to be C.

Maybe, with greater power of test (increasing dataset) some other significant epistatic interactions can be observed, since Barendse et al. (2007) reported additive-by-dominant interaction between CAPN4751 with SNPs on the CAST gene. It is very important to remember that epistatic effects must be estimated jointly with marginal additive and dominance effects. When we remove the marginal effects, several epistatic interactions were highly significant but the estimates are contaminated with marginal effects.

In the near future, the genetic gain for tenderness using maker information of SNPs on CAST and CAPN1 genes can be expected for Nellore cattle. However, it is important to mention that the limiting factor may be the reduced percentage of residual variance explained by those markers, usually between 0.5 and $2 \%$. It is necessary to remember that quantitative traits are influenced by many genes, thus, it is the sum of effects that we should consider.

\section{CONCLUSION}

The markers on the CAPN1 gene (CAPN4751) and on the CAST gene (UOGCAST and WSUCAST) have additive and dominant effects on tenderness measured by Warner Bratzler shear force of meat with 7,14 and 21 days of aging in Nellore cattle. However, there are epistatic interactions between CAPN4751 and markers on the CAST gene that must be considered, because the favorable allele for CAPN4751 depends on the genotypic class of markers on the CAST gene. Also, the additive effect of the markers UOGCAST and WSUCAST is not observed when $C A P N 4751$ has the TT genotype. Improving the sample size may be necessary for new tests, because the CAPN4751 showed few animals with the CC genotype, which may have influenced the results with the epistatic model.

\section{ACKNOWLEDGMENTS}

The authors would like to thank FAPESP (Project \#07/50736-2) and Merial-Igenity for financial supports.

\section{REFERENCES}

Barendse W, Harrison BE, Hawken RJ, Ferguson DM, et al. (2007). Epistasis between calpain 1 and its inhibitor calpastatin within breeds of cattle. Genetics 176: 2601-2610.

Bonilha SFM, Tedeschi LO, Pachker IU, Razook AG, et al. (2008). Evaluation of carcass characteristics of Bos indicus and tropically adapted Bos taurus breeds selected for postweaning weight. J. Anim. Sci. 50: 841-851.

Casas E, White SN, Riley DG, Smith TP, et al. (2005). Assessment of single nucleotide polymorphisms in genes residing on chromosomes 14 and 29 for association with carcass composition traits in Bos indicus cattle. J. Anim. Sci. 83: 13-19.

Casas E, White SN, Wheeler TL, Shackelford SD, et al. (2006). Effects of calpastatin and micro-calpain markers in beef cattle on tenderness traits. J. Anim. Sci. 84: 520-525. 
Cheong HS, Yoon DH, Park BL, Kim LH, et al. (2008). A single nucleotide polymorphism in CAPN1 associated with marbling score in Korean cattle. BMC Genet. 9: 33.

Corva P, Soria L, Schor A, Villarreal E, et al. (2007). Association of CAPN1 and CAST gene polymorphisms with meat tenderness in Bos taurus beef cattle from Argentina. Genet. Mol. Biol. 30: 1064-1069.

Garcia MD, Michal JJ, Gaskins CT, Reeves JJ, et al. (2006). Significant association of the calpastatin gene with fertility and longevity in dairy cattle. Anim. Genet. 37: 304-305.

Koohmaraie M (1996). Biochemical factors regulating the toughening and tenderization process of meat. Meat Sci. 43: 193-201.

Morris CA, Cullen NG, Hickey SM, Dobbie PM, et al. (2006). Genotypic effects of calpain 1 and calpastatin on the tenderness of cooked M. longissimus dorsi steaks from Jersey x Limousin, Angus and Hereford-cross cattle. Anim. Genet. 37: 411-414.

Page BT, Casas E, Heaton MP, Cullen NG, et al. (2002). Evaluation of single-nucleotide polymorphisms in CAPN1 for association with meat tenderness in cattle. J. Anim. Sci. 80: 3077-3085.

Page BT, Casas E, Quaas RL, Thallman RM, et al. (2004). Association of markers in the bovine CAPN1 gene with meat tenderness in large crossbred populations that sample influential industry sires. J. Anim. Sci. 82: 3474-3481.

Pérez-Enciso M and Misztal I (2004). Qxpak: a versatile mixed model application for genetical genomics and QTL analyses. Bioinformatics 20: 2792-2798.

SAS (2004). SAS/STATUser's Guide: version 9.1. SAS Institute, North Caroline, 5136.

Schenkel FS, Miller SP, Jiang Z, Mandell IB, et al. (2006). Association of a single nucleotide polymorphism in the calpastatin gene with carcass and meat quality traits of beef cattle. J. Anim. Sci. 84: 291-299.

Smith TPL, Casas E, Rexroad CE III, Kappes SM, et al. (2000). Bovine CAPN1 maps to a region of BTA29 containing a quantitative trait locus for meat tenderness. J. Anim. Sci. 78: 2589-2594.

White SN, Casas E, Wheeler TL, Shackelford SD, et al. (2005). A new single nucleotide polymorphism in CAPN1 extends the current tenderness marker test to include cattle of Bos indicus, Bos taurus, and crossbred descent. J. Anim. Sci. 83: 2001-2008. 